\title{
Habilidades sociais e autoeficácia de médicos e enfermeiros: um estudo comparativo
}

\author{
Social skills and self-efficacy of doctors and nurses: a comparative \\ study \\ http://dx.doi.org/10.5007/2178-4582.2014v48n1p138
}

\author{
Jacqueline Maia de Miranda \\ Universidade Veiga de Almeida, Rio de Janeiro/RJ, Brasil \\ Adriana Benevides Soares \\ Universidade Salgado de Oliveira, Niterói/RJ, Brasil
}

\begin{abstract}
O objetivo do estudo foi comparar as habilidades sociais e a autoeficácia em médicos e enfermeiros uma vez que, historicamente, a prática médica tem sido centrada na autoridade do conhecimento e nas práticas mais curativas enquanto que a da enfermagem tem sido alicerçada no zelo do paciente e nas práticas mais paliativas. Participaram 168 indivíduos, sendo 82 médicos e 86 enfermeiros. Foram utilizados o Inventário de Habilidades Sociais, o Inventário de Empatia e a Escala de Autoeficácia Geral Percebida adaptada para a realidade brasileira. Os resultados obtidos mostraram que as habilidades sociais se relacionam com a autoeficácia e que apesar dos médicos terem apresentado maior número de correlações positivas entre habilidades sociais e autoeficácia, os enfermeiros apresentaram escores mais altos em habilidades sociais do que os médicos. Os resultados apresentaram ainda um escore semelhante em ambos os grupos para a variável autoeficácia.
\end{abstract}

Palavras-chave: Habilidades Sociais - Autoeficácia - Médicos - Enfermeiros.
The aim of the study was to compare the social skills and self-efficacy in doctors and nurses, since medical practice historically has been focused on the authority of knowledge and healing practices, while nursing has been founded on the zeal for the patient and also on merely palliative practices. The study included 168 individuals of which 82 were doctors and 86 nurses. It was used Social Skills Inventory, Inventory Empathy and the General Perceived Self-efficacy Scale adapted to Brazilian reality. The results showed that social skills are related to self-efficacy and that, despite the fact that doctors have a greater number of positive correlations between social skills and self-efficacy, nurses had higher scores in social skills than the formers. The results also showed similar scores in both groups in relation to the efficacy variable.

Keywords: Social Skills - Self-Efficacy - Doctors - Nurses.

\section{Introdução}

No mundo cada vez mais globalizado e, consequentemente, mais competitivo, observa-se que as relações sociais estão se tornando cada vez mais superficiais e as práticas de relacionamentos interpessoais eficazes têm sido exercidas com dificuldade. No entanto, há uma exigência por práticas mais competentes nos relacionamentos imposta pelo próprio sistema que gerencia toda essa diversidade. É o que vem ocorrendo em relação às práticas de atendimento de médicos e enfermeiros. 
Aos profissionais de saúde se apresentam demandas de desempenhos que exigem tanto a competência técnica (capacidade analítica e instrumental) quanto a social (DEL PRETTE; DEL PRETTE, 2003). Os profissionais que visam à promoção da saúde devem se apropriar da capacidade analítica e também da instrumental e da social para que o desempenho profissional possa ser mais eficiente, propiciando maior adesão, participação do paciente no tratamento e tendo, por consequência, um encurtamento da doença com o sucesso do tratamento (Ibid.).

A competência técnica vem, há muito tempo, sendo a única determinante no exercício das profissões de saúde. A competência social, entretanto, só mais recentemente passa a ser considerada como relevante, ainda que timidamente, para o desempenho eficaz da relação terapêutica. Além disso, é necessário que os principais atores dos serviços de saúde, médicos e enfermeiros, não só exerçam suas competências com destreza, mas também acreditem no exercício de suas próprias capacidades.

\section{Referencial teórico: habilidades sociais e autoeficácia}

As habilidades sociais são definidas por Del Prette e Del Prette (2011, p. 20) como sendo "classes de comportamentos sociais que somente podem ser classificadas como tais na medida em que contribuem para a competência social". Seja qual for o ambiente de trabalho, as relações interpessoais requerem dos indivíduos um acervo de habilidades e competências sociais com o propósito de um melhor desempenho interpessoal e laboral (Id., 2010). As relações interpessoais dos profissionais de saúde com os pacientes vêm muitas vezes se tornando restritas ao contato direto com a enfermidade apresentada (RIBEIRO; AMARAL, 2008).

O tratar da patologia tem sido o elemento exclusivo para o cumprimento das responsabilidades clínicas, deixando de se considerar o lado psicológico do ser humano (CAPRARA;RODRIGUES, 2004; GRAÇAS; SANTOS, 2009; RIBEIRO; AMARAL, 2008). O sistema de saúde legitima o saber técnico do médico e todo o seu conhecimento acadêmico, sem levar em consideração as relações interpessoais sugeridas pela literatura (KESTENBERG; FALCONE, 2011; DEL PRETTE;DEL PRETTE, 2003), como capazes de facilitar as intervenções e a adesão ao tratamento. No estudo de Ribeiro e Amaral (2008), por exemplo, pode-se identificar que em estudantes de medicina brasileiros as atitudes, ainda que mais centradas no paciente do que no médico quando se compara aos estudantes norte-americanos, são ainda bastante numerosas no que diz respeito às atitudes que focalizam o poder médico.

Como os serviços de saúde devem ser eficazes e efetivos, a sociedade legitima o médico com todo o seu saber, conhecimento e componentes técnicos adquiridos durante sua especialização para que obtenha êxito em sua prescrição. Enquanto o médico tem a função primordial de curar, o enfermeiro tem 
a de cuidar. Essas atribuições do médico fazem com que sejam bastante valorizadas na função médica a competência técnica, sendo admitidas, inclusive em nome deste saber, práticas autoritárias; enquanto na prática do enfermeiro é mais valorizada e tida como mais importante a competência relacional, sendo, neste caso, inadmissível comportamentos indelicados que não visem o bem-estar do paciente (CAPRARA; RODRIGUES, 2004; MIRANDA; VARGAS, 2009; VASCONCELLOS; SILVA, 2011).

$\mathrm{Na}$ realidade, tanto o enfermeiro quanto o médico necessitam ser dotados de uma competência tanto no quesito interpessoal quanto no técnico-acadêmico (LEADEBAL et al., 2009; STANISCIA et al., 2011). Essa dupla competência pode possibilitar mais humanização e eficácia na cura das doenças (GOMES et al., 2012). Com o propósito de melhorar a adesão ao tratamento clínico prescrito, bem como a adaptação ou aceitação de todo o processo patológico, o indivíduo deve se sentir cuidado, entendido como ser humano, tratado como sujeito, e não como doença (KOIFMAN, 2001; SELLI et al., 2008).

É natural que em uma sociedade cada vez mais exigente sejam requeridas competências ímpares dos médicos e enfermeiros. No decorrer dos anos esta premência tem se dado em função do tratar do indivíduo e na eficácia dos procedimentos e prescrições. Na atualidade, exige-se também um relacionamento interpessoal mais efetivo com esses profissionais (PIMENTEL et al., 2008). Considera-se o desenvolvimento de habilidades sociais como comportamento protetivo da saúde (JEFFERY, 1989; MURTA, 2005), sendo fundamental para amenizar situações de desconforto e auxílio nas situações que envolvam os direitos humanos básicos (MURTA, 2005).

Furtado, Falcone e Clark (2003) consideram que o déficit das habilidades sociais pode acarretar o desenvolvimento de estresse, e que o desempenho acadêmico pode sofrer em decorrência de situações estressoras na medida em que há também a deficiência do repertório de habilidades sociais. O estudo mostrou que a graduação em Medicina valoriza o conhecimento técnico e dá muita ênfase às exigências acerca das questões teórico-práticas, supondo que somente estratégias de desenvolvimento destes conhecimentos são suficientes para proporcionar, ao aluno, uma maneira eficaz de desempenhar suas atribuições concernentes do cuidado ao ser humano.

É indiscutível que a competência técnica é fundamental em ambas as profissões, porém, nem sempre as competências sociais são devidamente valoradas em sua relevância. No estudo realizado por Stein e Kwan (1999) em que analisaram as reclamações dos usuários de um plano de saúde, pode-se detectar que a maior insatisfação relatada consistia nas habilidades de relacionamento médico-paciente em sua estrutura de comunicação verbal. O estudo proporcionou a realização de workshops para o desenvolvimento das habilidades necessárias de comunicação e foi percebida, com a realização do trabalho, uma melhora nesta habilidade, proporcionando, assim, uma maior satisfação dos usuários. 
No estudo de Barros, Falcone e Pinho (2011) foi também possível verificar a carência de comportamentos empáticos por parte do médico em relação ao paciente tanto na rede pública de saúde quanto na privada. Também Kestenberg e Falcone (2011), após a realização de um treinamento da habilidade empática com enfermeiros, evidenciaram que a capacidade de compreender e interpretar os pacientes são atributos indispensáveis na enfermagem uma vez que o paciente é capaz de regular sua afetividade e permitir que processos de autopreservação sejam construídos.

Profissionais que apresentam "déficits" em suas habilidades sociais podem sofrer desconforto e insatisfação em suas relações interpessoais, uma vez que pode deixar de ocorrer uma reciprocidade, equilíbrio e troca, acarretando um convívio pouco saudável (DEL PRETTE; DEL PRETTE, 2011). Da mesma forma, deficiências nas habilidades sociais empáticas podem gerar problemas nas relações pessoais e profissionais, uma vez que o distanciamento da dor do outro, que se apresenta muitas vezes com o formato de autoproteção, pode ser interpretada como descaso (KESTENBERG et al., 2006; KESTENBERG e FALCONE, 2012).

Além do desenvolvimento das habilidades relacionais, é preciso que tanto médicos quanto enfermeiros pensem que são capazes de desempenhar estes comportamentos técnicos, sociais e emocionais de forma competente. Para isso, é preciso que acreditem em sua eficácia pessoal e construam um sistema de crenças que os faça confiar em suas próprias habilidades pessoais.

Bandura (1982) sustenta que o mecanismo de autoeficácia está centralizado na autopercepção de eficácia e que cada indivíduo é capaz de influenciar suas próprias ações, seus pensamentos e emoções. O mesmo autor (1994) identifica que as pessoas são capazes de criar e desenvolver percepções pessoais sobre si mesmas utilizando mecanismos que as levem a alcançar os objetivos traçados, bem como o controle do ambiente onde estão inseridas. Enfatiza que os indivíduos não são agentes cognitivos intencionais e que os processos causais determinam a motivação e a ação das pessoas.

Os indivíduos operacionalizam os estímulos, de forma autônoma, mecânica e emergente, ou seja, um comportamento oriundo de estruturas mais complexas derivadas das inúmeras relações interpessoais (BANDURA, 1989). A autoeficácia percebida é capaz de ajudar a explicar diversos fenômenos, tais como as mudanças no comportamento de enfrentamento; os níveis de reações fisiológicas de estresse; a autorregulação do comportamento; a resignação e o desânimo diante a experiências ligadas ao fracasso; os efeitos de descontrole; a ilusão da ineficácia; a luta por realizações; o crescimento do interesse intrínseco; e as buscas profissionais (BANDURA, 1982).

Bandura (1988; 1989) diferencia o termo "eficácia", definido por ele como a capacidade de lidar com o ambiente, de "autoeficácia" entendido como o julgamento da pessoa sobre sua capacidade de se organizar e executar atitudes para atingir certo padrão de desempenho. Schultz e Schultz (2009) 
definem autoeficácia como a percepção que o indivíduo tem de sua autoestima e da competência em lidar com os problemas da vida. Myers (2000) acrescenta ainda que pessoas com uma alta capacidade de autoeficácia apresentam maior persistência, menor ansiedade e são menos deprimidos, conquistando, assim, um estado mais elevado de saúde e sucesso na vida acadêmica e profissional.

Bandura (1989) sustenta que nada é mais central ou persuasivo do que as crenças das pessoas sobre suas capacidades de exercerem domínio acerca dos eventos que afetam suas vidas. Para Myers (2000), as pessoas que apresentam um forte senso de autoeficácia se apresentam mais centradas diante de situações que possam trazer conflitos no cotidiano, uma vez que essa capacidade traduz a persistência, a calma e a elaboração de novas estratégias de solução.

Rabelo e Cardoso (2007) afirmam que a expectativa da autoeficácia na teoria cognitiva é fator determinante para definir um comportamento inicial, bem como a demanda que é aplicada a ele, ou seja, a maneira como o interlocutor verbaliza ou exprime suas necessidades e o tempo de sua duração. No entanto, na busca constante de sucesso, como também do conhecimento dos limites humanos, o homem é capaz de desempenhar comportamentos necessários na obtenção de suas conquistas e de seus desejos (BARREIRA e NAKAMURA, 2006). Os mecanismos utilizados, que visam ao refinamento dos processos emocionais, cognitivos ou motivacionais voltados para a melhoria das capacidades e habilidades comportamentais, podem também proporcionar mudanças nos ambientes sociais deste indivíduo (PAJAREZ e OLAZ, 2008); ou seja, na qualidade do pensamento crítico face às falhas de julgamentos que afetam o desempenho (BANDURA, 1989).

Bandura (Ibid.) mostra que o indivíduo possui uma capacidade para perceber aspectos positivos ou negativos e, de acordo com sua autoeficácia percebida, norteia seu desempenho. De acordo com Pajares e Olaz (2008), a teoria social cognitiva propõe que os indivíduos eficazes são pessoas auto-organizadas, proativas, autorreflexivas, e autorreguladas e que, por meio de uma dinâmica de inter-relação, se moldam e se orientam pelas exigências e influências ambientais que ocorrem em situações de relacionamento. As crenças de autoeficácia são capazes de alterar o funcionamento cognitivo sob a influência das operações motivacionais (BANDURA, 1989).

Alguns poucos estudos têm sido realizados para mostrar características de autoeficácia dos profissionais de saúde. Rebelo (2008), em estudo sobre a autoeficácia percebida pelos fisioterapeutas acerca de seu desempenho profissional, revelou um elevado índice de percepção de autoeficácia por estes profissionais. Para Gago (2008), a percepção de autoeficácia nos papéis de carreira dos enfermeiros com formação pós-graduada se manifesta mais intensamente na importância atribuída ao papel de estudante e de trabalhador e demonstram mais importância nos valores de Realização, Promoção, Estético e de Autoridade. Por outro lado, os enfermeiros mais novos e sem experiência 
apresentam crenças de autoeficácia mais elevadas na realização de atividades em tempos livre. Homens e mulheres manifestam um nível semelhante de crenças, enquanto trabalhadores e nos seus valores relacionados com a realização.

Tendo em vista a literatura apresentada e a importância de se estudar as competências relacionais nos profissionais de saúde, este estudo objetivou identificar e relacionar habilidades sociais e crenças de autoeficácia de médicos e enfermeiros comparando as duas classes profissionais.

\section{Método}

\section{Participantes}

A amostra contou com 81 homens $(48,2 \%)$ e 87 mulheres $(51,8 \%)$. Do total de 168 avaliados, 82 pertenciam à formação em Medicina (48,8\%), sendo que, neste grupo, 41 participantes eram do gênero masculino e 41 do gênero feminino. Da formação em Enfermagem, o total foi de 86 participantes $(51,2 \%)$, sendo 40 do gênero masculino e 46 do gênero feminino. A idade média foi de 38,1 anos (desvio padrão $=12,4$ ). Tanto médicos quanto enfermeiros possuíam nível superior completo, eram graduados há pelo menos um ano e trabalhavam em hospitais.

\section{Instrumentos}

Foram utilizados os seguintes instrumentos: Inventário de Habilidades Sociais - Del Prette e Del Prette (2001), Inventário de Empatia IE - Falcone, Ferreira, Luz, Fernandes, Faria, D`Augustin, Sardinha, e Pinho (2008) e a Escala de Autoeficácia Geral Percebida (Schwarzer e Jerusalem, 1995, adaptada para a realidade brasileira por Souza e Souza (2004).

\section{Inventário de Habilidades Sociais - IHS}

O IHS-Del Prette (2001) é um inventário de autorrelato composto por 38 questões de situações e reações em que o respondente avalia a frequência com que reage de acordo com uma escala de cinco itens. A escala utilizada é a do tipo Likert com 5 pontos que variam de nunca ou raramente a sempre ou quase sempre, devendo o respondente marcar a frequência com que reage a cada situação expressa em cada item. Parte dos itens é redigida de modo negativo, ou seja, com frequência mais elevada na reação que indica déficit na habilidade relacionada àquela situação. $\mathrm{O}$ inventário avalia cinco fatores específicos, são eles: F1 $(\alpha=0,96)$ - habilidades de enfrentamento com risco (11 itens); F2 $(\alpha=0,86)$ - habilidade de autoafirmação na expressão do afeto positivo (7 itens); F3 $(\alpha=0,81)$ - habilidades de conversação e desenvoltura social (7 itens); F4 $(\alpha=0,75)$ - habilidades de autoexposição a desconhecidos ou a 
situações novas ( 3 itens); F5 ( $\alpha=0,74)$ - habilidades de autocontrole da agressividade (3 itens). O escore total permite a primeira avaliação dos recursos e déficits do sujeito, enquanto os fatoriais revelam os subgrupos específicos nos quais o sujeito apresenta um bom repertório ou déficits. Há 7 itens que não se relacionam com nenhum fator, mas que são mantidos por serem considerados no escore total.

\section{Inventário de Empatia - IE}

O inventário (FALCONE et al., 2008) é composto por 40 itens que envolvem 16 situações sociais em que o indivíduo deve responder com que frequência as afirmações se aplicam a si próprios, de acordo com uma escala tipo Likert que apresenta frases que expressam uma posição claramente favorável e uma posição claramente desfavorável. O inventário avalia quatro fatores: F1 $(\alpha=0,85)$ - Tomada de Perspectiva - caracteriza-se por uma capacidade de entender a perspectiva e sentimentos da outra pessoa (12 itens); F2 ( $\alpha=$ 0,77); Flexibilidade Interpessoal - caracteriza-se por uma elevada capacidade em aceitar perspectivas, crenças e valores muito diferentes, além de tolerância à frustração provocada pela atitude do interlocutor (10 itens); F3 $(\alpha=0,74)$; Altruísmo - caracteriza-se por uma capacidade de sacrificar temporariamente as próprias necessidades, em benefício de outra pessoa (9 itens); F4 ( $\alpha=0,72$ ); Sensibilidade Afetiva - é a tendência a experimentar sentimentos de compaixão e de interesse pelo estado emocional da outra pessoa (9 itens).

O construto empatia foi medido pelo Inventário de Empatia (Falcone et al., 2008); no entanto, entende-se que este conceito está incluído no repertório de habilidades sociais. O referido instrumento foi utilizado, pois apresenta de maneira mais consistente os fatores ligados à tomada de perspectiva, flexibilidade, altruísmo e sensibilidade afetiva, fatores significativos para este estudo.

\section{Escala de Autoeficácia Geral Percebida}

Trata-se de uma escala do tipo Likert, com dez itens que variam de 1 a 5. Quanto maior a pontuação maior a percepção de autoeficácia. Cada item refere-se ao alcance de metas e insinua uma atribuição interna estável de sucesso. A escala original (SCHWARZER; JERUSALÉM, 1995) apresenta um Alpha de Cronbach de 0,84, além de estar correlacionada positivamente com o otimismo e autoestima e, negativamente, com a ansiedade, depressão e pessimismo. A Escala de Autoeficácia Geral Percebida, adaptada para a realidade brasileira (SOUZA; SOUZA, 2004) apresenta Alpha de Cronbach de 0,81.

\section{Procedimentos de coleta de dados}

Foram aplicados os três instrumentos precedidos do questionário de levantamento dos dados demográficos de forma individual. Houve uma explicação prévia de como responder aos instrumentos. Não foi estipulado tempo 
determinado para responder a nenhum dos três instrumentos. Os questionários foram preenchidos em papel e foram recolhidos posteriormente.

\section{Procedimentos éticos}

Com o objetivo de documentar as Diretrizes e Normas Regulamentadoras de pesquisa envolvendo seres humanos dispostos na resolução 196/96 do Conselho Nacional de Saúde (CNS), o presente estudo sob n ${ }^{\circ}$ 115/2011 foi encaminhado ao Comitê de Ética que aprovou sua aplicação em 08 de fevereiro de 2011. Aos participantes desse projeto foi solicitada a assinatura do Termo de Consentimento Livre e Esclarecido.

\section{Procedimentos de análise de dados}

Para avaliar a relação entre os fatores dos instrumentos e entre instrumentos foram realizadas correlações de Pearson. Foram realizados teste $t$ de Student para verificar as diferenças entre os grupos.

\section{Apresentação dos resultados}

Para avaliar as hipóteses apresentadas nessa pesquisa, inicialmente efetuou-se a análise descritiva. Com o propósito de avaliar a relação entre as habilidades sociais e a autoeficácia utilizou-se o teste de correlação linear de Pearson, com nível de significância de $\mathrm{p}<0,05$.

$\mathrm{Na}$ amostra total, a autoeficácia apresentou correlação positiva significativa com todas as variáveis de habilidades sociais variando de 0,16 a 0,41 , exceto com a variável Empatia - fator Sensibilidade Afetiva, cuja correlação não foi significativa, o que indica que os indivíduos que participaram da pesquisa apresentam forte crença na própria capacidade de exercer controle sobre eventos para alcançar as metas desejadas, ou seja, possuem valores elevados de autoeficácia. Na amostra de médicos, a autoeficácia apresentou correlação positiva significativa com todas as variáveis de habilidades sociais variando de 0,24 a 0,48, exceto com os fatores Autocontrole da Agressividade e fator Sensibilidade Afetiva cujas correlações não foram significativas. $\mathrm{Na}$ amostra de enfermeiros, a autoeficácia apresentou correlação positiva significativa com a metade das variáveis de habilidades sociais variando de 0,23 a 0,37 , exceto com os fatores Autoafirmação, Conversação e Desenvoltura Social, Tomada de Perspectiva, Flexibilidade Interpessoal e Sensibilidade Afetiva cujas correlações não foram significativas.

Em geral, na amostra de médicos verificou-se mais correlações significativas e mais fortes entre autoeficácia e habilidades sociais que na amostra de enfermeiros. A exceção é quanto ao fator Autocontrole da Agressividade cuja correlação com a autoeficácia não foi significativa entre os médicos, mas foi entre os enfermeiros. Os resultados podem ser observados na Tabela 1. 
Tabela 1. Correlação da Autoeficácia com as Habilidades Sociais e Empatia

\begin{tabular}{|c|c|c|c|c|c|c|}
\hline \multirow{2}{*}{$\begin{array}{c}\text { Habilidades Sociais e } \\
\text { Empatia }\end{array}$} & \multicolumn{2}{|c|}{$\begin{array}{c}\text { Autoeficácia } \\
\text { Amostra Total }\end{array}$} & \multicolumn{2}{|c|}{$\begin{array}{l}\text { Autoeficácia } \\
\text { Médicos }\end{array}$} & \multicolumn{2}{|c|}{$\begin{array}{l}\text { Autoeficácia } \\
\text { Enfermeiros }\end{array}$} \\
\hline & $\mathbf{r}$ & $\mathbf{p}$ & $\mathbf{R}$ & $\mathbf{p}$ & $\mathbf{r}$ & $\mathbf{p}$ \\
\hline Fator Enfrentamento & $0,37^{* *}$ & 0,00 & $0,45^{* * *}$ & 0,00 & $0,34^{* *}$ & 0,00 \\
\hline Fator Autoafirmação & $0,20^{* *}$ & 0,00 & $0,32^{* *}$ & 0,00 & 0,14 & 0,20 \\
\hline $\begin{array}{l}\text { Fator Conversação e } \\
\text { desenvoltura social }\end{array}$ & $0,18^{*}$ & 0,01 & $0,29^{* *}$ & 0,00 & 0,12 & 0,27 \\
\hline $\begin{array}{l}\text { Fator Autoexposição } \\
\text { a desconhecidos }\end{array}$ & $0,27^{* *}$ & 0,00 & $0,33^{* *}$ & 0,00 & $0,27^{*}$ & 0,01 \\
\hline $\begin{array}{l}\text { Fator Autocontrole } \\
\text { da agressividade }\end{array}$ & $0,22^{* *}$ & 0,00 & 0,17 & 0,12 & $0,27^{*}$ & 0,01 \\
\hline IHS_Total & $0,41^{* *}$ & 0,00 & $0,48^{* * * *}$ & 0,00 & $0,37^{* *}$ & 0,00 \\
\hline $\begin{array}{l}\text { Fator Tomada de } \\
\text { Perspectiva }\end{array}$ & $0,18^{*}$ & 0,01 & $0,25^{*}$ & 0,02 & 0,16 & 0,13 \\
\hline $\begin{array}{l}\text { Fator Flexibilidade } \\
\text { Interpessoal }\end{array}$ & $0,16^{*}$ & 0,03 & $0,24^{*}$ & 0,02 & 0,13 & 0,20 \\
\hline Fator Altruísmo & $0,25^{* *}$ & 0,00 & $0,30^{* *}$ & 0,00 & $0,23^{*}$ & 0,02 \\
\hline $\begin{array}{l}\text { Fator Sensibilidade } \\
\text { Afetiva }\end{array}$ & 0,07 & 0,34 & 0,00 & 0,94 & 0,10 & 0,31 \\
\hline
\end{tabular}

Para verificar as diferenças entre o grupo de médicos e o grupo de enfermeiros, nos construtos habilidades sociais e autoeficácia foi realizado o teste $t$, conforme se expõe na Tabela 2. Os fatores que apresentaram diferenças significativas foram os de Enfrentamento, Autoexposição a desconhecidos para o grupo dos enfermeiros. Os fatores: Tomada de Perspectiva e Flexibilidade Interpessoal, também apresentaram diferenças significativas, indicando que enfermeiros, nestes fatores, são mais empáticos do que médicos. No construto autoeficácia não houve diferença significativa entre médicos e enfermeiros conforme apontado na Tabela 2. 
Tabela 2. Comparação de grupos para Habilidades Sociais, Empatia e Autoeficácia

\begin{tabular}{|c|c|c|c|c|c|}
\hline & Formação & $\mathbf{M}$ & DP & $\mathbf{t}$ & $\mathbf{p}$ \\
\hline \multirow{2}{*}{$\begin{array}{l}\text { Fator } \\
\text { Enfrentamento }\end{array}$} & Medicina & 9,44 & 3,05 & $-2,21$ & $0,02 *$ \\
\hline & Enfermagem & 10,57 & 3,38 & & \\
\hline \multirow{2}{*}{ Fator Autoafirmação } & Medicina & 9,90 & 1,27 & 1,44 & 0,15 \\
\hline & Enfermagem & 9,57 & 1,59 & & \\
\hline \multirow{2}{*}{$\begin{array}{l}\text { Fator Conversação } \\
\text { e desenvoltura } \\
\text { social }\end{array}$} & Medicina & 7,02 & 1,76 & 0,66 & 0,50 \\
\hline & Enfermagem & 6,82 & 2,06 & & \\
\hline \multirow{2}{*}{$\begin{array}{l}\text { Fator } \\
\text { Autoexposição a } \\
\text { desconhecidos }\end{array}$} & Medicina & 3,02 & 1,38 & $-3,14$ & 0,00 \\
\hline & Enfermagem & 3,64 & 1,15 & & \\
\hline \multirow{2}{*}{$\begin{array}{l}\text { Fator Autocontrole } \\
\text { da agressividade }\end{array}$} & Medicina & 3,17 & 0,79 & $-0,53$ & 0,59 \\
\hline & Enfermagem & 3,23 & 0,72 & & \\
\hline \multirow{2}{*}{ IHS_Total } & Medicina & 32,61 & 5,78 & $-1,52$ & 0,13 \\
\hline & Enfermagem & 34,04 & 5,77 & & \\
\hline \multirow{2}{*}{$\begin{array}{l}\text { Fator Tomada de } \\
\text { Perspectiva }\end{array}$} & Medicina & 3,61 & 0,54 & $-2,00$ & 0,04 \\
\hline & Enfermagem & 3,78 & 0,56 & & \\
\hline \multirow{2}{*}{$\begin{array}{l}\text { Fator Flexibilidade } \\
\text { Interpessoal }\end{array}$} & Medicina & 3,01 & 0,58 & $-2,74$ & 0,00 \\
\hline & Enfermagem & 3,28 & 0,68 & & \\
\hline \multirow{2}{*}{$\begin{array}{l}\text { Fator Fator } \\
\text { Altruísmo }\end{array}$} & Medicina & 2,90 & 0,51 & $-1,68$ & 0,09 \\
\hline & Enfermagem & 3,05 & 0,64 & & \\
\hline \multirow{2}{*}{$\begin{array}{l}\text { Fator Sensibilidade } \\
\text { Afetiva }\end{array}$} & Medicina & 4,10 & 0,51 & 1,01 & 0,31 \\
\hline & Enfermagem & 4,02 & 0,55 & & \\
\hline \multirow{2}{*}{ AE_Geral } & Medicina & 4,05 & 0,50 & 0,62 & 0,53 \\
\hline & Enfermagem & 3,99 & 0,68 & & \\
\hline
\end{tabular}

Fonte: Tabela elaborada pelos autores 


\section{Discussão dos resultados}

Os resultados apresentados neste estudo indicam que indivíduos que apresentam maior repertório de habilidades sociais apresentam também maior percepção de autoeficácia, corroborando o que diz Bandura (1988), ou seja, quanto mais competente for o desempenho, maior é a capacidade do indivíduo em perceber-se capaz em realizar um comportamento competente.

A necessidade desses profissionais da saúde, ao longo de suas vidas fez provavelmente com que tivessem desenvolvido habilidades de comunicação, de civilidade, de assertividade, de empatia, de trabalho e de expressão de sentimentos positivos, isto é, habilidades indispensáveis para atender com maior eficácia o outro. Assim, exercendo com competência seus comportamentos relacionais, puderam também se perceber mais autoeficazes em suas funções. Pôde-se observar que, quanto mais o sujeito reconhece o papel que desempenha, maior é sua confiança no enfrentamento das situações cotidianas aversivas. Foi possível observar, ainda, maior número de correlações significativas no grupo de médicos do que no grupo de enfermeiros. Possivelmente, a vivência diária do grupo de médicos pode ter colaborado para que tenham adquirido, ao longo de sua prática, as habilidades necessárias ao exercício competente e, consequentemente, um bom desempenho laboral; afinal, a prática é uma maneira de desenvolver competências (MELLO, 2010).

Quanto à comparação entre médicos e enfermeiros no que diz respeito a habilidades sociais, apesar dos médicos terem apresentado maior número de correlações positivas entre habilidades sociais e autoeficácia, os enfermeiros apresentaram escores mais altos de habilidades sociais do que os médicos. No grupo dos enfermeiros, os escores mais elevados foram apresentados nos fatores Enfrentamento com Risco e Autoexposição a Desconhecidos. Os enfermeiros apresentam maior competência social nesses comportamentos. As pessoas realizam um mecanismo de análise de suas próprias ações e são capazes também de realizarem intervenções motivacionais, afetivas e cognitivas acerca de seus comportamentos e atitudes (KESTENBERG; FALCONE, 2011).

Possivelmente, na amostra de enfermeiros, o escore do fator Enfrentamento com Risco se apresentou com maior pontuação, pois é o enfermeiro quem lida primeiramente com as demandas que se apresentam no contexto hospitalar. É justamente ele que tem a incumbência de receber o paciente, que se apresenta na maioria das vezes ansioso e debilitado, manifestando muitas vezes comportamentos agressivos. Cabe ao enfermeiro, tranquilizar o paciente a fim de que a intervenção possa ser realizada e para que ocorra uma adesão ao tratamento, ajudando, assim, na melhoria do quadro. Já no fator Autoexposição a Desconhecidos, o traquejo social é fundamental, uma vez que envolve componentes das habilidades de conversação na abordagem do outro que não lhe é familiar. Stein e Kwan (1999) também demonstraram a importância do desenvolvimento de melhores habilidades de conversação em seu estudo. 
Quanto aos resultados apresentados no inventário de empatia, os enfermeiros apresentaram diferenças significativas nos fatores Tomada de Perspectiva e Flexibilidade Interpessoal. $\mathrm{O}$ que caracteriza, para esses profissionais, uma capacidade de entender e aceitar a perspectiva de outra pessoa, seus valores, atitudes e sentimentos, além de uma tolerância à frustração. Provavelmente, os enfermeiros adquiriram, ao logo de seu trabalho, a possibilidade de escutar o outro, de uma maneira peculiar, se colocando em seu lugar e vivenciando, de maneira mais empática, todo o sofrimento que demanda o ambiente hospitalar. Esse sujeito escuta as dores e sentimentos de indivíduos em sofrimento e com eles passam a maior parte do tempo. Essa situação, proporciona ao enfermeiro maior envolvimento e, consequentemente, o desenvolvimento das habilidades necessárias à escuta e a empatia (KESTENBERG et al, 2006; KESTENBERG; FALCONE, 2011, KESTENBERG; FALCONE, 2012).

\section{Considerações finais}

Este estudo objetivou identificar e comparar habilidades sociais e autoeficácia de médicos e enfermeiros. Os resultados obtidos na pesquisa embora apresentem limitações - uma vez que a amostra foi restrita - mostraram que as habilidades sociais se relacionam com a autoeficácia; que, nos fatores do IHS, os médicos apresentaram maior número de correlações significativas com a variável autoeficácia, e os enfermeiros apresentaram escores mais altos de habilidades sociais do que os médicos.

Estudos indicam que o desenvolvimento das habilidades sociais necessárias para o desempenho das práticas profissionais de saúde são importantes nos contextos de atendimentos ambulatoriais, hospitalares e clínicos. A adesão ao tratamento, bem como a mobilização emocional podem colaborar consideravelmente para a ativação de fatores acerca da melhoria do estado de saúde dos pacientes.

Foi possível compreender que o julgamento que o indivíduo faz de suas atitudes e de seu comportamento é elemento importante na construção da crença de autoeficácia e que os problemas relativos ao ambiente hospitalar - o sofrimento, a doença a relação médico-paciente - são cercados de componentes estressantes e aversivos que poderiam ser melhor vivenciados com desenvolvimento de habilidades sociais.

Vale a pena considerar que os resultados obtidos são importantes para que as políticas públicas que envolvem o Programa Único de Saúde definam rumos de acordo com as necessidades específicas de cada região brasileira e que os Projetos Políticos Pedagógicos estejam imbuídos de fomentar nos jovens universitários o desejo de fazer a diferença na vida de outra pessoa, uma vez que os Conselhos Federativos determinam e priorizam o respeito e o direito do ser humano em sua totalidade. 
A partir desse estudo podem-se realizar novas pesquisas acerca dos atendimentos e como os usuários percebem que médicos e enfermeiros estabelecem as relações interpessoais no momento em que se faz necessário maior integração e harmonia; ou seja, no momento crítico de seu estado de saúde, quando o paciente se torna mais vulnerável, mais perceptivo e, muitas vezes, até agressivo. Como perspectivas futuras outros estudos podem promover a avaliação desses profissionais pelos seus pacientes, e que médicos possam ser avaliados por enfermeiros, bem como os enfermeiros possam avaliar os médicos.

Com os resultados obtidos, deseja-se reforçar a relevância de que os cursos de formação de médicos e enfermeiros adotem medidas educacionais e estratégias que proporcionem ao estudante um desenvolvimento das habilidades sociais tão necessárias ao contexto hospitalar, aumentando a autoeficácia desses profissionais e corroborando, assim, a determinação da LDB 9394/96 que sugere o desenvolvimento das habilidades e competências do estudante.

\section{Referências bibliográficas}

BANDURA, Albert. Self-efficacy mechanism in human agency. American psychologist, [S.1.] v. 37, n.2, p.122-147, 1982.

. Organizational Applications of Social Cognitive Theory. Australian

Journal of Management. [S.I], The University of New South Wales, v.13, n.2, p.275-302, 1988.

. Human agency in social cognitive theory. American Psychologist,[S.1.], v.44. n.9, p.1175-1184, 1989.

.Self-efficacy. In: RAMACHAUDRAN, Vilayanur Subramanian . Encyclopedia of human behavior, v.4, p.71-81, 1994.

BARREIRA, Diná Dornelles.; NAKAMURA, Antonieta Pepe. Resiliência e autoeficácia percebida: articulação entre conceitos. Aletheia, [S.1.], v.23, p.75-80, 2006.

BARROS, Patrícia de Sousa.; FALCONE, Eliane Mary de Oliveira; PINHO, Vanessa Dordron. Avaliação da empatia médica na percepção de médicos e pacientes em contextos público e privado de saúde. Arq. ciênc. saúde, [S.1.], v.18, n.1, p.36-43, 2011.

CAPRARA, Andrea; RODRIGUES, Josiane. A relação assimétrica médico-paciente: repensando o vínculo terapêutico. Ciênc. saúde coletiva, v.9, n.1, p.139-46, 2004.

DEL PRETTE, Zilda. A. P. Inventário de Habilidades Sociais (IHS-Del-Prette): Manual de aplicação, apuração e interpretação. São Paulo: Casa do Psicólogo, v.1, p.70, 2001.

DEL PRETTE, Almir.; DEL PRETTE, Zilda Aparecida Pereira. No contexto da travessia para o ambiente de trabalho: treinamento de habilidades sociais com universitários. Estudos de Psicologia, Natal, v.8, n.3, p.413-420, 2003. 
.Psicologia das relações interpessoais: vivências para o trabalho em grupo. 8 ed. Petrópolis: Vozes, 2010. 2011.

.Habilidades sociais: intervenções efetivas em grupo. São Paulo: Casa do Psicólogo,

FALCONE, Eliane Mary Oliveira. et al. Inventário de Empatia (IE): Desenvolvimento e validação de uma medida brasileira. Avaliação Psicológica,[S.1.], v.7, n.3, p.321-334, 2008.

FURTADO, Eliane de Sousa. et al. Avaliação do estresse e das habilidades sociais na experiência acadêmica de estudantes de medicina de uma universidade do Rio de Janeiro. Interação em Psicologia,,Curitiba, v.7, n.2, 2003.

GAGO, Tânia. Crenças de eficácia nos papéis de carreira, valores de vida e prática de supervisão de ensinos clínicos, em enfermeiros. 2008, 229 fls. Dissertação (Mestrado em Ciências da Educação),Universidade de Lisboa, Lisboa, 2008.

GOMES, Annatalia Meneses de Amorim Gomes. et al. Relação médico-paciente: entre o desejável e o possível na Atenção Primária à Saúde. Physis: Revista de Saúde Coletiva, Rio de Janeiro, v.22, n.3, 2012.

GRAÇAS, Elizabeth Mendes; SANTOS, Geralda Fortina. Metodologia do cuidar em enfermagem na abordagem fenomenológica. Revista da Escola de Enfermagem da USP, São Paulo, v.43, n.1, p. 200-207, 2009.

JEFFERY, Robert W. Risk behaviors and health: Contrasting individual and population perspectives. American Psychologist, [S.1.], v.44, n.9, p.1194, 1989.

KESTENBERG, Célia Caldeira Fonseca. et al. Cuidando do estudante e ensinando relações de cuidado de enfermagem. Texto Contexto em Enfermagem, Florianópolis, v.15, n. esp, 2006.

KESTENBERG, Célia Caldeira Fonseca; FALCONE, Eliane Mary de Oliveira. Programa de promoção da empatia para graduandos em enfermagem. In: DEL PRETTE, Zilda. A. P; DEL PRETTE, Almir. (Org.). Habilidades Sociais: Intervenções efetivas em grupo. São Paulo: Casa do Psicólogo, 2011. p.115-144.

KESTENBERG, Célia Caldeira Fonseca, FALCONE, Eliane Mary de Oliveira. Empatia em enfermeiros: a importância da tomada de perspectiva na redução da angústia pessoal. In: FALCONE, Eliane M. de Oliveira; OLIVA, Angela Donato; FIGUEIREDO, Cristiane (Orgs.). Produções em terapia cognitivo-comportamental. São Paulo: Casa do Psicólogo, 2012. p.71-79.

KOIFMAN, Lilian. O modelo biomédico e a reformulação do currículo médico da Universidade Federal Fluminense. História, Ciências, Saúde-Manguinhos, [S.1.], v.8, n.1, p.49-69, 2001.

LEADEBAL, Oriana Deyze C. Paiva. et al. Análise das bases didático-pedagógicas para o ensino da sistematização da assistência enfermagem. Revista Mineira de Enfermagem, Belo Horizonte, v.13, n. 1, p.65-75, 2009.

MELLO, Thatiana Valory dos Santos. Habilidades sociais, empatia e frustação em graduandos de medicina. 2010. 135fls. Dissertação (Mestrado em Psicologia) - Universidade Salgado de Oliveira, Niterói, 2010. 
MIRANDA, J. M.; SOARES, A. B. Habilidades sociais e autoeficácia de médicos e enfermeiros: um...

MIRANDA, Seara Pereira.; VARGAS, Divane. Satisfação de pacientes de um centro de atenção psicossocial álcool e drogas com o atendimento do enfermeiro. SMAD. Revista eletrônica saúde mental álcool e drogas, Ribeirão Preto, v.5, n.2, p.1-15, 2009.

MURTA, Sheila Giardini. Aplicações do treinamento em habilidades sociais: análise da produção nacional. Psicologia: Reflexão e Crítica, Porto Alegre, v.18, n.2, p.283-291, 2005.

MYERS, David. G. Psicología Social. 2.ed. Rio de Janeiro: LTC, 2000.

PAJARES, Frank; OLAZ Fabián. O. Teoria Social Cognitiva e Autoeficácia: uma visão geral. In: BANDURA, Albert.; AZZI, Roberta Gurgel; POLYDORO, Soely. (Org.), Teoria Social Cognitiva: conceitos básicos, Porto Alegre: Artmed, 2008, p. 97-114,

PIMENTEL, Naiene Santos. et al. Comportamento verbal na prática clínica: considerações sobre o operante na análise do comportamento. In: SILVA, Wander C. M. Pereira. Sobre o comportamento e cognição: análise comportamental aplicada, v.21, 2008, p.45-60.

REBELO, Pedro Jorge Lapa. Estudo exploratório sobre as atitudes de profissionais das saúde face a eficácia da fisioterapia e dos fisioterapeutas e sua relação com a autoeficácia percebida pelos fisioterapeutas. 2008, 149 fls. Dissertação (Mestrado em Comunicação em Saúde) - Universidade Aberta, Lisboa, 2008.

RIBEIRO, Maria Mônica. Freitas; AMARAL, Carlos Faria Santos. Medicina centrada no paciente e ensino médico: a importância do cuidado com a pessoa e o poder médico. Revista Brasileira de Educação Médica, Rio de Janeiro, v.32, n.1, p.90-7, 2008.

SELLI, Lucilda. et al. O cuidado na ressignificação da vida diante da doença. $O$ Mundo da Saúde. [S.1.], v.32, n.1, p. 85-90, 2008.

SOUZA, Israel; SOUZA, Marcos Aguiar. Validação da escala de autoeficácia geral percebida. Revista universidade rural: série ciências humanas, Rio de Janeiro, v.26, n.1-2, p.12-17, 2004.

SCHULTZ, Duane P.; SCHULTZ, Sydney Hellen. História da Psicologia Moderna. 9.ed. São Paulo: Cengage Learning, 2009.

STANISCIA, Ana Carolina M. et al. Dificuldades emocionais vivenciadas pelos médicos intensivistas da unidade de terapia - adulto de um hospital geral privado. Revista da Sociedade Brasileira de Psicologia Hospitalar, [S.1.],v.14, n.1, p.41-73, 2011.

STEIN, Terry S.; KWAN, Julie. Thriving in a busy practice: physician-patient communication training. Effective clinical practice: ECP, [S.1.], v.2, n.2, p.63-70, 1998.

VASCONCELLOS Sheyna Cruz; SILVA, Borges Humildes Cruz. Um estudo psicológico sobre a interação do auxiliar de enfermagem e o paciente crônico. Revista da Sociedade Brasileira de Psicologia Hospitalar, [S.1.], v.14, n.2, p.112-141, 2011.

Submissão em: 06/05/2013

Revisão em: 29/07/2013

Aceite em: 31/07/2013 
Jacqueline Maia de Miranda é professora de Pedagogia da Universidade Veiga de Almeida e do Centro Universitário Fluminense. Pedagoga do Instituto Dom Bosco Colégio Salesiano Campos. Desenvolve projetos na área de Orientação Profissional. Estudante do décimo período de Psicologia no Institutos Superiores de Ensino do Colégio Nossa Senhora Auxiliadora. Endereço para correspondência: Estrada de Perynas, s/nº - CEP 28901-970, Rio de Janeiro/RJ, Brasil. E-mail: jacmiada@hotmail.com

Adriana Benevides Soares é doutora em Psicologia Cognitiva pela Université de Paris Sud, França (1995). Professora titular da Universidade Salgado de Oliveira, Niterói/RJ, Brasil. E-mail: adribenevides@gmail.com 